\title{
Comment by the author
}

The reader has expressed his concerns about the surgical technique investigated in our study, in which the results of K-wire arthrodesis and coracoclavicular augmentation were retrospectively evaluated [3]. The technique was described first by Murray in 1940 and Phemister in 1942. Since then, 150 different surgical procedures have been described for the treatment of Tossy type III injuries. However, the management of acromioclavicular separations remains challenging to this day. Recent recommendations include an individualized approach to these injuries, with indications for non-operative and surgical therapy based on the type of injury (Tossy/Rockwood classification) and patients' characteristics [1].

Indeed, complications such as migration or breakage of K-wires used at the shoulder can be associated with severe sequelae, which have been described several times in the literature $[1,2]$. In our study, minor complications associated with $\mathrm{K}$-wire migration in the lateral direction with the risk of skin penetration were detected in two cases. In both of these patients, uneventful removal was performed under local anaesthesia without any additional consequences for the subsequent postoperative course or clinical outcome. From our point of view, bending by $180^{\circ}$ and intraosseous fixation of the lateral end of $\mathrm{K}$-wires into the acromion considerably reduces the risk of medial migration. In addition, using K-wires of an adequate size (at least $2.0 \mathrm{~mm}$ ) makes breakage improbable. Nevertheless, the implantation of K-wires around the shoulder should be restricted to compliant patients-and should be avoided for athletes who intend to participate in contact sports or who are at risk of sequel injuries. However, for these patients, conservative management is already advisable because the complication rate is high, varying from 42 to $58 \%$, irregardless of the surgical procedure [1].

As mentioned by the reader, the principles of surgical treatment of acromioclavicular joint (ACJ) separations include ACJ reduction and the prevention of acute re-displacement $[2,5]$. However, even direct ligament repair or ligament substitution, as suggested by Weaver and Dunn, requires sufficient protection of soft tissue repair. Coracoclavicular screws, hook plates and K-wires can be used for this purpose. To date, none of these devices has been shown to be superior to any of the others, and all still require early removal to prevent shoulder stiffness. Results of arthroscopically assisted procedures are encouraging, but clinical experiences of these techniques are still being in the development stage [4].

The retrospective nature of the study accounts for the comparatively high drop-out rate. Nevertheless, the reader's assumption is speculative that those 32 patients who did not follow up may still have their $\mathrm{K}$-wires in situ. Rather, it is more likely that K-wire removal was performed elsewhere and not in our outpatient clinic. Removal is easy to perform under local anaesthesia, which represents an advantage of $\mathrm{K}$-wires over other protectors of soft tissue repair.

Overall, our results support coracoclavicular augmentation together with temporary K-wire arthrodesis for the treatment of ACJ separations. Nevertheless, when $\mathrm{K}$-wires are used around the shoulder, possible complications, including severe sequelae of K-wire migration, ought to be considered. If strict patient compliance cannot be provided, implants with more stability, such as hook plates, are advised for soft tissue protection.

Tim T. Lögters

Düsseldorf, Germany

e-mail:Tim.Loegters@uni-duesseldorf.de

\section{References}

1. Clarke HD, McCann PD. Acromioclavicular joint injuries. Orthop Clin North 2000;31:177-187.

2. Fraser-Moodie JA, Shortt NL, Robinson CM. Injuries to the acromioclavicular joint. J Bone Jt Surg 2008;90B:697-707.

3. Lögters TT, Briem D, Lohde C, Janssen A, Rueger JM, Windolf J, Linhart W. K-wire arthrodesis and coracoclavicular augmentation of complete acromioclavicular separations: functional and subjective results. Eur J Trauma Emerg Surg 2008;34:43-48.

4. Norell H Jr, Llewllyn RC. Migration of a threaded Steinmann pin from an acromioclavicular joint into the spinal canal: a case report. J Bone Jt Surg 1965;47A:1024-1026.

5. Taft TN, Wilson FC, Oglesby JW. Dislocation of the acromioclavicular joint. An end result study. J Bone Jt Surg 1987;69A:1045-1051. 